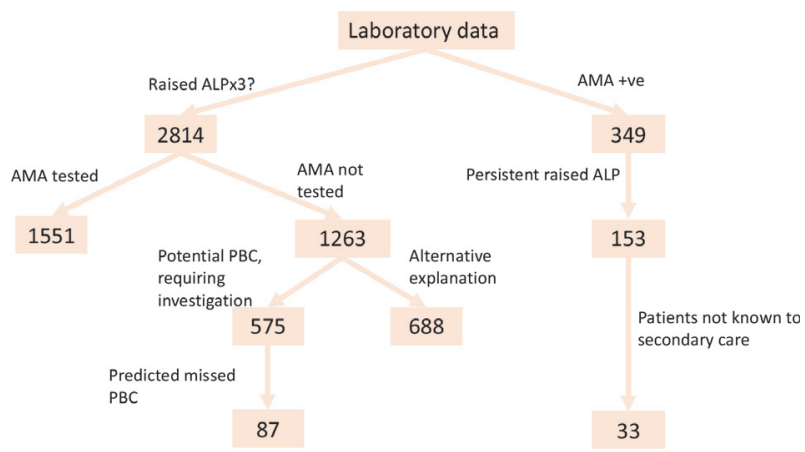

Abstract PTH-40 Figure 1

raised ALP with no other explanation who had AMA tested, $15.2 \%$ were AMA + ve. Applying this to our group with no AMA or alternative explanation for raised ALP, 87 cases of PBC may have been missed with only $46.6 \%$ of patients with PBC appropriately investigated and diagnosed. Separately 349 AMA +ve patients were identified. $35.0 \%$ had a normal ALP, $14.3 \%$ had a intermittently/borderline raised ALP, and $44 \%$ had a persistently raised ALP; of these $21.9 \%$ were not known to secondary care. [Figure 1]

Conclusions From data on individuals with previous LFTs we estimate that $>50 \%$ of patients with PBC in Somerset remain undiagnosed, and over 20\% meeting diagnostic criteria of PBC were not referred. These data show that for patients who have had LFTs performed, analysis of laboratory data and a 'case-finding' approach could be effective in reducing liver disease related harm.

\section{PTH-41 OUTCOMES OF THE MODIFIED PATHWAY FOR URGENT UPPER GI CANCER REFERRALS DURING THE COVID-19 PANDEMIC}

Sardar Chaudhary*, Mairi McLean, Perminder Phull. Aberdeen Royal Infirmary, Aberdeen, UK

\subsection{6/gutjnl-2021-BSG.356}

Introduction There has been significant change in endoscopy referral pathways as departments reorganise their services during COVID-19. As per BSG guidance, a modified referral pathway was instituted locally for patients with urgent suspicion of upper gastrointestinal cancer (USOC-UGI). We aimed to assess the impact of this modified pathway on outcomes of USOC-UGI referrals during the deceleration and early recovery phases of endoscopy provision during COVID-19 at our institution.

Methods We performed a retrospective observational singlecentre study, comparing outcomes of the COVID-impacted USOC-UGI pathway from March to June 2020 to the corresponding pre-COVID period in 2019 when all patients had straight to test endoscopy. During the COVID-impacted period all patients referred were first assessed in a virtual clinic (VC) by a consultant gastroenterologist. Risk stratification and the Edinburgh dysphagia score (EDS) questionnaire were used to triage patients; alternative investigations such as barium study and CT requested when indicated. The primary outcome was cancer detection rate. We also assessed vetting outcomes, symptom concordance, and delayed cancer diagnosis at 6month follow-up.
Results 253 patients were included in the study. 118 patients were referred through the modified COVID-impacted pathway and 135 in 2019, indicating a drop in referrals by $12.5 \%$ $(\mathrm{p}=0.13)$. The median age was similar $(66.5 \mathrm{yrs}$ in COVID impacted $v s 67 \mathrm{yrs}$ pre-COVID). The most common reason for referral during both periods was dysphagia $(58.5 \%$ vs $62.9 \%$; $\mathrm{P}=0.221$ ). Symptom concordance between those recorded in the referral and those reported at the time of $\mathrm{VC}$ was $86.4 \%$. Following VC, 27/118 (22.9\%) patients were deemed not to require UGI investigations of which $9(7.6 \%)$ stated that their symptoms had resolved. Nearly half $(55.3 \%)$ of the patients reviewed in $\mathrm{VC}$ were referred for an alternative investigation to endoscopy, of which barium swallow and meal was most common 36/57 (63\%). Overall, the rate of upper GI cancer diagnosed between both periods was not statistically different (8.5\% vs 5.2\%; $\mathrm{P}=0.3$ ). All patients diagnosed with oesophageal cancer reported to have symptoms of dysphagia and had a calculated EDS of $\geq 5$. There has been no delayed cancer diagnosis at 6-month follow-up.

Conclusions The rate of UGI cancer was similar between the two time periods. Initial virtual consultation reduced the demand for endoscopy or alternative investigations by nearly a quarter. Dysphagia remains an especially worrisome symptom and the incorporation of EDS questionnaire in this pathway appears appropriate. The use of alternative investigations in a select group of patients during restricted endoscopy services seems safe although long-term follow-up data is still required.

\section{PTH-42 UTILISING A FRAILTY SCORING TOOL IMPROVES THE APPROPRIATENESS OF EMERGENCY GI BLEED ENDOSCOPY}

Andrew Claridge*. Great Western Hospital, swindon, UK

\subsection{6/gutjnl-2021-BSG.357}

Introduction Risk assessment tools have been used for over 20 years to determine the need and timeliness of endoscopic intervention in patients with symptoms of acute upper gastrointestinal bleed (AUGIB), but with easier access to procedures and an increasingly frail population being referred for emergency and aggressive management the addition of a clinical frailty score (CFS) to existing pathways should be considered. This study looks at the patient factors leading to the emergency gastroscopy (OGD), co-morbidity and survival.

Methods Clinical, endoscopic and outcome data were collected for all adult patients referred for emergency AUGIB gastroscopy during a 12 month period. Frailty was scored by the endoscopist using the 9 point Rockwood CFS (Rockwood, et al., 2005). Follow up data were collected until death or for a minimum 1 year and analysed using GraphPad Prism version 8.0.0, USA.

Results 143 patients underwent OGD in line with NICE CG141 guidance. Survivors were followed up for an average 85 weeks (range 53-126). Of the 143 patients, 90 (63\%) were males, the median age 73 years and 41 (29\%) patients $>80$ years old. In total $17(12 \%)$ died during admission, 15 $(11 \%)<4$ weeks, $23(16 \%)<12$ weeks and $44(31 \%)<52$ weeks. $56(39 \%)$ patients in total died during follow up.

11 patients had Blatchford score $\leq 2$, average age 38 years. One patient passed away at week 39 of unrelated illness and no AUGIB at OGD. All scores $\leq 2$ had coexisting non-GI issues contributing to their presentation. 


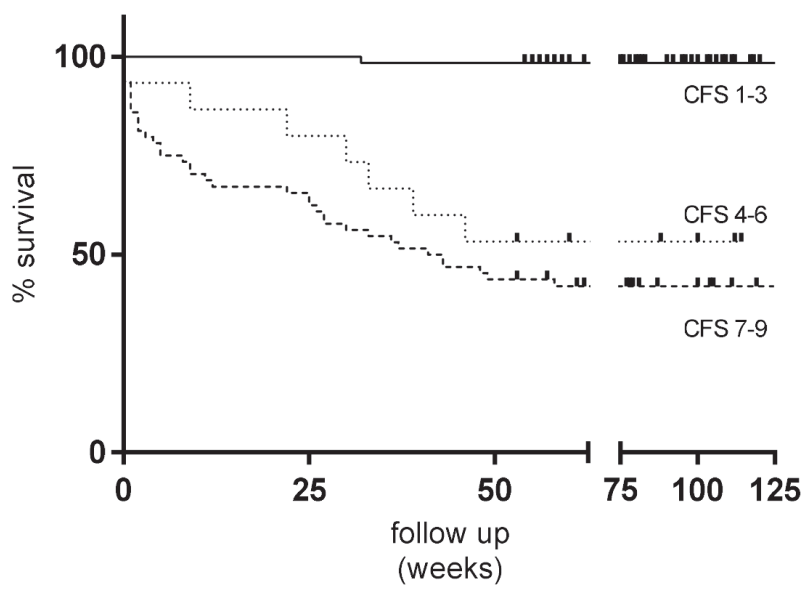

Abstract PTH-42 Figure 1 Kaplan-Meier curves of survival, grouped according to the CFS

Although Blatchford scores $>2$ were not a predictor of early outcome or the use of intervention, there is a correlation between Blatchford score and death over time. The Blatchford score is significantly greater in those with survival $<4$ weeks (14.6) compared with those $<52$ weeks (8.9, $\mathrm{p}<0.01)$. Likewise pre and post Rockall score is correlated with 52 week survival, ( $\log$ rank test, $\mathrm{p}<0.01$ ), but a poor indicator of the need to intervene at OGD, or death from GI causes. Indicating that both risk assessment scores are in fact surrogate markers of frailty rather than GI pathology.

All patients that died during admission had a CFS 7-9, and of the $15(11 \%)$ patients that died $<4$ weeks $80 \%$ had CFS 7-9. Of the 23 patients who died $<12$ weeks $91 \%$ had a CFS 7-9. Only $1 / 64(1.5 \%)$ of patients with a CFS $1-3$ survived $<52$ weeks.

Conclusion A Blatchford score $\leq 2$ is strongly predictive of no AUGIB.

No scoring system predicts reliably whether intervention was required. A high percentage of those patients with poor outcomes during admission, and $<12$ weeks have a CFS of 7 9 , in contrast to the excellent long term survival of those patients with a CFS 1-3.

The Rockwood CFS can help differentiate those patients who may have poor outcomes from undertaking emergency OGD, particularly in the short term, giving an opportunity to discuss and pursue a more conservative approach.

\section{PTH-43 IMPROVING ENDOSCOPY TURNAROUND TIME AND LIST EFFICIENCY THROUGH IMPLEMENTATION OF A MULTI- FACETED IMPROVEMENT PLAN}

${ }^{1}$ Karishma Sethi Arora*, ${ }^{2}$ Chehkuan Tai, 'Laura Marelli. 'Homerton University Hospital, London, UK; ${ }^{2}$ The Royal Free Hospital, London, UK

\subsection{6/gutjnl-2021-BSG.358}

Introduction Maximising efficiency in endoscopy in the face of increasing service pressure, demand and complexity of endoscopy is imperative, particularly given the unparalleled impact of the COVID pandemic on service delivery and cancer pathways. Previous attempts to improve turnaround time through introduction of a turnaround nurse have been hindered by inadequate staffing. We aimed to improve list efficiency and improve turnaround time through the application of marginal gains theory and implementation of a multi-faceted improvement plan.

Methods Data was collected from electronic patient records and audit entries made by endoscopy staff. List 'actual' start and stop times were audited against 'scheduled' times. Turnaround time was assessed by a Quality Improvement (QI) Fellow, observing the endoscopy list and collecting information in real time. Results were discussed at a departmental meeting and a 4-stage improvement plan was devised and implemented. Re-audit data was collected to determine effect.

Results Preliminary audit data revealed $89 \%$ of list starts to be delayed. Lists ran beyond scheduled stop times in $73 \%$. The improvement plan saw:

1) Review and re-organisation of the nursing rota.

2) Departmental commissioning of an external 'change agent' to conduct interviews with nursing and endoscopy managers and work with the QI fellow in designing a bespoke team-building day to address communication strategies and brainstorm perceived departmental issues hindering efficiency.

3) Introduction of an in-room debrief tool, to enhance situation awareness and teamwork.

4) Implementation of a multi-modal 'Nurse-led consent' training programme, combining didactic and in-situ scenariobased simulation training, measuring and recording competence using Directly-Observed-Procedural-Skills (DOPS) assessments.

Re-audit data revealed improved list finish-times (49\% cf. $27 \%$ ) although delays in start-times remained. Median turnaround time was 10 minutes, a major improvement from a turnaround time in 20.8 minutes in 2018.

Conclusions It is recognised that single improvement interventions are unlikely to result in significant, sustainable change. The aggregation of marginal gains theory dictates that small, marginal gains can add up to a remarkable improvement. Our 4-stage improvement plan saw the implementation of a revised nursing rota and a bespoke team-building day in tandem with the introduction of a 'Nurse Consent' training programme and a novel team debrief tool. In this way, we were able to implement change, whilst simultaneously assessing and addressing staff morale, engage key stakeholders and as a result significantly improve turnaround time. We plan to streamline admission and patient preparation processes to further address delayed start times in future cycles of the improvement project.

\section{PTH-44 IMPROVING THE MORECAMBE BAY ACUTE UPPER GASTROINTESTINAL BLEEDING SERVICE}

Christopher Mysko*, Rami Mohamed, Ahmed Hamdy, Firas Elfourtia, John Keating. University Hospitals Morecambe Bay NHS Foundation Trust, UK

\subsection{6/gutjnl-2021-BSG.359}

Introduction Acute upper gastrointestinal bleeding (AUGIB) is a common medical emergency with an estimated incidence of 134 per 100,000 population; 1 presentation every 6 minutes. Mortality is high at approximately $10 \%$ and is largely unchanged over the last two decades with UK-wide audits revealing variability and poor care standards despite the development of guidelines. The British Society of Gastroenterology (BSG) recently published the 'AUGIB Care Bundle' to address deficiencies and drive sustained improvements. ${ }^{1}$ 